\title{
Numerical Technique for Solution and Error Estimate for the Initial Value Problem
}

\author{
By W. E. Simon
}

1. Introduction. Numerical techniques for the solution of initial value differential equations whose solutions are well behaved are well established. However, if the solutions are known to have movable poles, certain computational difficulties arise. One of the most straightforward and simple numerical techniques is that of continuous analytic continuation, which is simply an extension of the solution from point to point with a truncated Taylor series. Unfortunately, the rate of convergence of the Taylor series goes to zero as a pole is approached. In addition, a good estimate of the error of the solution is difficult to obtain.

2. Analysis. Consider the initial value problem

$$
\begin{aligned}
& y^{k}=f\left(x, y, y^{\prime}, \cdots, y^{k-1}\right), \\
& y^{l}\left(x_{0}\right)=y_{0}^{l} \\
& {[l=0,1,2, \cdots,(k-1)] .}
\end{aligned}
$$

Now $y^{l}$ may be expanded in a truncated Taylor series about any nonsingular point, $x_{p}$, in the form

$$
y_{n}{ }^{l}\left(x_{p+1}\right)=\sum_{i=0}^{n-l} \frac{y_{n}^{(i+l)}\left(x_{p}\right)\left[x_{p+1}-x_{p}\right]^{i}}{i !} \quad[l=0,1,2, \cdots,(k-1)]
$$

A sequence of $x$ 's may be chosen and $y^{l}$ computed for each of these points in succession by use of Eq. (2). Thus the technique of continuous analytic continuation. A primary difficulty with this technique, if constant increments of $x$ are used, is that the rate of convergence of Eq. (2) is a function of $x$. In particular, if $y(x)$ exhibits poles, Eq. (2) does not converge at all at these poles. In order to circumvent this difficulty for regions distant from a pole, we make the following requirement:

$$
\left|\frac{y^{i}\left(x_{p}\right)\left[x_{p+1}-x_{p}\right]^{i}}{i !}\right| \leqq \frac{\epsilon^{i}}{i !} \quad(i=1,2, \cdots, n) .
$$

We now choose $x_{p+1}$ so that Eq. (3) is satisfied, where $\epsilon$ is some arbitrarily small, positive number. This ensures that the truncation error is uniformly small in $x$.

As the sequence $x_{p}$ approaches a pole of the solution, $\left(x_{p+1}-x_{p}\right) \rightarrow 0$ and $\left|y\left(x_{p+1}\right)\right| \rightarrow \infty$. Using either an upper bound on $\left|y\left(x_{p+1}\right)\right|$ or a lower bound on $\left|x_{p+1}-x_{p}\right|$, a point $x_{q}$ is chosen at which the continuous analytic continuation is halted and the solution resumed as a truncated Laurent series about the pole. The $k$ number of unknown coefficients in the Laurent series is determined from $y_{n}{ }^{l}\left(x_{q}\right)$ $[l=0,1,2, \cdots(k-1)]$. Note that one of these unknown coefficients is necessarily the location of the pole. After the pole is passed and the criterion for using the Laurent series is no longer satisfied, the continuous analytic continuation is again picked up and continues as before.

Received April 29, 1964. 
A second difficulty with this procedure is the unknown error associated with the choice of $n$ and, $\epsilon_{d}$. To estimate this error, let us compute a second solution, $y_{n-1}$, where the new truncated Taylor series will be

$$
y_{n-1}^{l}\left(x_{p+1}\right)=\sum_{i=0}^{n-l-1} \frac{y_{n-1}^{(i+l)}\left(x_{p}\right)\left[x_{p+1}-x_{p}\right]^{i}}{i !} \quad[l=0,1,2, \cdots,(k-1)] .
$$

Now consider the difference between the two approximate solutions. If the limit of the approximate solution approaches the solution of the differential equation as $n \rightarrow \infty$, Eq. (5), then it follows that the difference between two approximate solutions is the order-of-magnitude of their error, if $n$ is sufficiently large, Eq. (6).

$$
\begin{aligned}
\lim _{n \rightarrow \infty} y_{n}\left(x_{p+1}\right) & =y\left(x_{p+1}\right) . \\
y\left(x_{p+1}\right)-y_{n}\left(x_{p+1}\right) & =O\left[y_{n}\left(x_{p+1}\right)-y_{n-1}\left(x_{p+1}\right)\right] .
\end{aligned}
$$

Thus we can obtain a numerical solution to the initial value problem and an associated estimate of the error. Note that since the determination of the coefficients of the Laurent series is dependent on $y^{l}\left(x_{q}\right)$, the error in the location of the pole of $y(x)$ causes a pole in the error of $y_{n}(x)$. In spite of this, the error in regions at a small distance from the poles of $y(x)$ may be quite satisfactory.

3. Application. The differential equation chosen for the application of this technique is the first transcendent equation of Painlevé in its canonical form, Eq. (7).

$$
\begin{aligned}
& y^{\prime \prime}=6 y^{2}+\lambda x, \\
& x_{0}=0, \quad y\left(x_{0}\right)=1, \quad y^{\prime}\left(x_{0}\right)=0 .
\end{aligned}
$$

The solution of this equation is also the solution of a much larger set of equations that can be generated by the transformation

$$
\begin{aligned}
& z=\frac{a+b y}{c+d y}, \quad a d-b c \neq 0, \\
& u=u(x) .
\end{aligned}
$$

Eq. (7) is particularly useful for a test case, since if the parameter $\lambda$ is set to zero, the solution is periodic with period 2.4286508 and poles occur at 1.2143254 $+m \cdot 2.4286508(m=0,1,2, \cdots)$, where the numbers are good to the last figure. According to [1], the first pole occurs at 1.2068 for $\lambda=1$ and at 1.179 for $\lambda=5$. The error of these determinations is not specified. In addition, a Taylor's series solution for $\lambda=5, x=0.1$, has been used to determine $y=1.031141446$, correct to the last place.

A program was written for a small digital computer, IBM-1620, to obtain the solution of Eq. (7). A value of 6 was taken for $n$, and $\epsilon$ was specified as input data.

The truncated Laurent series used in the neighborhood of a pole is given by Eq. (8), where $x^{i}$ is the location of the $i$ th pole.

$$
\begin{aligned}
y=\frac{1}{\left(x-x^{i}\right)^{2}}+\frac{0}{\left(x-x^{i}\right)}+0 & +0 \cdot\left(x-x^{i}\right)-\frac{\lambda x^{i}\left(x-x^{i}\right)^{2}}{10} \\
& -\frac{\lambda\left(x-x^{i}\right)^{8}}{6}+h\left(x-x^{i}\right)^{4}+0 \cdot\left(x-x^{i}\right)^{5} .
\end{aligned}
$$


Note that since the differential equation is second order, all except two of the constants in the Laurent series may be determined by substitution into the differential equation. The two undetermined constants are just " $x$ ", the location of the pole about which the expansion is performed, and " $h$," the coefficient of $\left(x-x^{i}\right)^{4}$. The " $\lambda$ " which appears in some of the terms is just the parameter of the differential equation. Thus Eq. (8) and its first derivative, evaluated at $x_{q}$, the point at which the continuous analytic continuation is halted, provide two equations for the two unknown constants, " $x$ " and " $h$." These equations are solved numerically for the values of the two constants.

Eq. (8) was used whenever $|y|>10$. Tabulated results for $x, y$, and $y^{\prime}$ are given in Tables 1, 2, and 3 for $\epsilon=0.1$ and for $\lambda=0,1$, and 5. Comparison of computed locations of poles is given in Table 4.

A plot of the error estimate and the actual error is given in Figure 1 for the

TABLE 1

Numerical Solution of First Painlevé Equation for $\lambda=0(\epsilon=0.1)$

\begin{tabular}{|c|c|c|}
\hline$x$ & $y$ & $y^{\prime}$ \\
\hline $\begin{array}{l}0.0 \\
0.1 \\
0.2 \\
0.3 \\
0.4 \\
0.5 \\
0.6 \\
0.7 \\
0.8 \\
0.9 \\
1.0 \\
1.1 \\
1.2 \\
1.3 \\
1.4 \\
1.5 \\
1.6 \\
1.7 \\
1.8 \\
1.9 \\
2.0 \\
2.1 \\
2.2 \\
2.3 \\
2.4 \\
2.5 \\
2.6 \\
2.7 \\
2.8 \\
2.9 \\
3.0\end{array}$ & $\begin{array}{c}1.0 \\
1.0303028 \pm 1 \\
1.1249984 \pm 3 \\
1.2966686 \pm 5 \\
1.571030 \pm 1 \\
1.997030 \pm 2 \\
2.670095 \pm 4 \\
3.790274 \pm 8 \\
5.82948 \pm 2 \\
10.12278 \pm 4 \\
21.7699 \pm 1 \\
76.5088 \pm 8 \\
4872.6 \pm 4 \\
136.238 \pm 2 \\
29.0068 \pm 2 \\
12.25439 \pm 5 \\
6.72609 \pm 2 \\
4.24741 \pm 1 \\
2.932152 \pm 7 \\
2.158607 \pm 5 \\
1.674589 \pm 5 \\
1.363221 \pm 5 \\
1.165527 \pm 6 \\
1.050540 \pm 7 \\
1.002539 \pm 9 \\
1.01546 \pm 1 \\
1.09090 \pm 2 \\
1.23865 \pm 2 \\
1.47998 \pm 3 \\
1.85563 \pm 5 \\
2.44402 \pm 7\end{array}$ & $\begin{array}{r}0.0 \\
0.612182 \pm 1 \\
1.302034 \pm 3 \\
2.172699 \pm 5 \\
3.392648 \pm 8 \\
5.42110 \pm 2 \\
8.49381 \pm 3 \\
14.62214 \pm 5 \\
28.0786 \pm 1 \\
64.3828 \pm 4 \\
203.140 \pm 2 \\
1338.43 \pm 2 \\
680260 \pm \pm 90 \\
-3180.38 \pm 7 \\
-312.442 \pm 3 \\
-85.7728 \pm 5 \\
-34.8305 \pm 2 \\
-17.39252 \pm 6 \\
-9.84053 \pm 2 \\
-6.0193 \pm 1 \\
-3.844853 \pm 2 \\
-2.476392 \pm 4 \\
-1.52718 \pm 1 \\
-0.79791 \pm 2 \\
-0.17192 \pm 3 \\
0.43286 \pm 4 \\
1.09175 \pm 5 \\
1.89753 \pm 8 \\
2.9943 \pm 1 \\
4.6430 \pm 2 \\
7.3752 \pm 3\end{array}$ \\
\hline
\end{tabular}

* When decimal point is not given, error estimate applies to last figure of the primary number. 
TABLE 2

Numerical Solution of First Painlevé Equation for $\lambda=1(\epsilon=0.1)$

\begin{tabular}{|c|c|c|}
\hline$x$ & $y$ & $y^{\prime}$ \\
\hline $\begin{array}{l}0.0 \\
0.1 \\
0.2 \\
0.3 \\
0.4 \\
0.5 \\
0.6 \\
0.7 \\
0.8 \\
0.9 \\
1.0 \\
1.1 \\
1.2 \\
1.3 \\
1.4 \\
1.5 \\
1.6 \\
1.7 \\
1.8 \\
1.9 \\
2.0 \\
2.1 \\
2.2 \\
2.3 \\
2.4 \\
2.5 \\
2.6 \\
2.7 \\
2.8 \\
2.9 \\
3.0 \\
3.1 \\
3.2 \\
3.3 \\
3.4 \\
3.5 \\
3.6 \\
3.7 \\
3.8 \\
3.9 \\
4.0 \\
4.1 \\
4.2 \\
4.3 \\
4.4 \\
4.5 \\
4.6 \\
4.7 \\
4.8 \\
4.9\end{array}$ & $\begin{array}{c}1.0 \\
1.0304705 \pm 1 \\
1.1263659 \pm 1 \\
1.3014527 \pm 5 \\
1.583054 \pm 1 \\
2.022771 \pm 2 \\
2.721242 \pm 4 \\
3.890886 \pm 8 \\
6.03834 \pm 2 \\
10.62261 \pm 3 \\
23.3936 \pm 1 \\
87.7732 \pm 8 \\
22031 . \pm 3 \\
114.969 \pm 1 \\
26.7678 \pm 1 \\
11.61357 \pm 4 \\
6.43931 \pm 2 \\
4.06596 \pm 1 \\
2.77520 \pm 1 \\
1.98846 \pm 1 \\
1.46727 \pm 2 \\
1.09940 \pm 2 \\
0.82710 \pm 3 \\
0.61893 \pm 5 \\
0.45738 \pm 6 \\
0.33274 \pm 8 \\
0.2400 \pm 1 \\
0.1768 \pm 1 \\
0.1426 \pm 2 \\
0.1376 \pm 2 \\
0.1628 \pm 2 \\
0.2197 \pm 3 \\
0.3106 \pm 3 \\
0.4396 \pm 4 \\
0.6135 \pm 5 \\
0.8448 \pm 6 \\
1.1553 \pm 7 \\
1.584 \pm 1 \\
2.207 \pm 2 \\
3.174 \pm 2 \\
4.820 \pm 4 \\
8.028 \pm 9 \\
15.74 \pm 2 \\
43.3 \pm 1 \\
371 . \pm 3 \\
433 . \pm 3 \\
45.6 \pm 1 \\
16.21 \pm 3 \\
8.190 \pm 9 \\
4.874 \pm 4\end{array}$ & 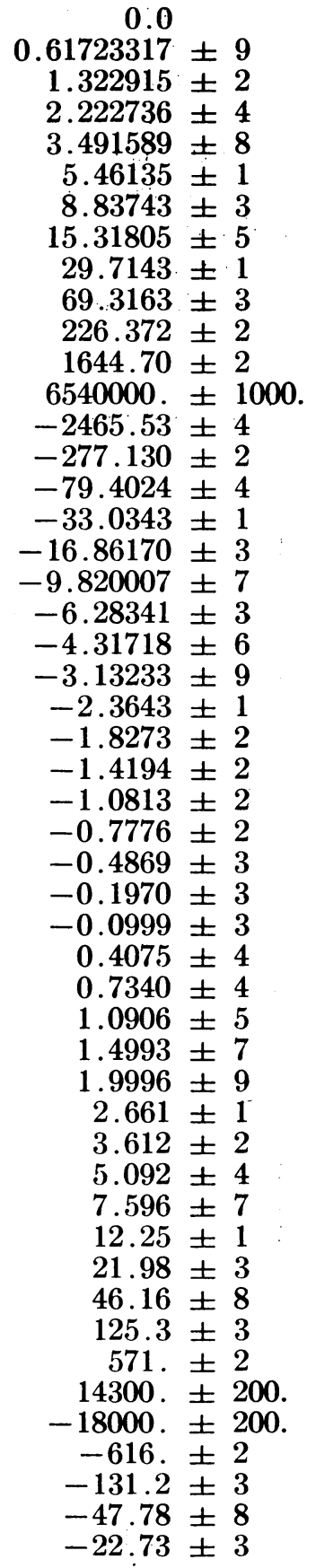 \\
\hline
\end{tabular}


TABLE 2-Continued

\begin{tabular}{l|r|r}
\hline$x$ & \multicolumn{1}{|c}{$y$} & \multicolumn{1}{c}{$y^{\prime}$} \\
\hline 5.0 & $3.166 \pm 2$ & $-12.78 \pm 1$ \\
5.1 & $2.148 \pm 2$ & $-8.114 \pm 6$ \\
5.2 & $1.471 \pm 1$ & $-5.656 \pm 4$ \\
5.3 & $0.9825 \pm 8$ & $-4.233 \pm 2$ \\
5.4 & $0.6078 \pm 6$ & $-3.319 \pm 2$ \\
5.5 & $0.3108 \pm 5$ & $-2.646 \pm 1$ \\
5.6 & $0.0754 \pm 4$ & $-2.067 \pm 1$ \\
5.7 & $-0.1031 \pm 2$ & $-1.500 \pm 1$ \\
5.8 & $-0.2238 \pm 1$ & $-0.908 \pm 1$ \\
5.9 & $-0.283530 \pm 3$ & $-0.282 \pm 1$ \\
6.0 & $-0.2796 \pm 1$ & $0.362 \pm 1$ \\
6.1 & $-0.2112 \pm 2$ & $1.005 \pm 1$ \\
6.2 & $-0.0079 \pm 4$ & $1.634 \pm 1$ \\
6.3 & $0.1156 \pm 5$ & $2.261 \pm 1$ \\
6.4 & $0.3746 \pm 6$ & $2.934 \pm 1$ \\
6.5 & $0.7073 \pm 7$ & $3.756 \pm 2$ \\
\hline
\end{tabular}

TABLE 3

Numerical Solution of First Painlevé Equation for $\lambda=5(\epsilon=0.1)$

\begin{tabular}{|c|c|c|}
\hline$x$ & $y$ & $y^{\prime}$ \\
\hline $\begin{array}{l}0.0 \\
0.1 \\
0.2 \\
0.3 \\
0.4 \\
0.5 \\
0.6 \\
0.7 \\
0.8 \\
0.9 \\
1.0 \\
1.1 \\
1.2 \\
1.3 \\
1.4 \\
1.5 \\
1.6 \\
1.7 \\
1.8 \\
1.9 \\
2.0 \\
2.1 \\
2.2 \\
2.3 \\
2.4 \\
2.5 \\
2.6 \\
2.7 \\
2.8 \\
2.9 \\
3.0\end{array}$ & $\begin{array}{r}1.0 \\
1.03114135 \pm 1 \\
1.1318367 \pm 3 \\
1.3205939 \pm 7 \\
1.631197 \pm 1 \\
2.125995 \pm 2 \\
2.927444 \pm 5 \\
4.300645 \pm 9 \\
6.90629 \pm 2 \\
12.78504 \pm 5 \\
31.0712 \pm 2 \\
158.785 \pm 2 \\
2346.9 \pm 1 \\
68.6973 \pm 6 \\
20.50311 \pm 9 \\
9.63692 \pm 3 \\
5.48114 \pm 1 \\
3.404024 \pm 6 \\
2.160451 \pm 2 \\
1.305679 \pm 2 \\
0.656339 \pm 7 \\
0.13706 \pm 1 \\
-0.27377 \pm 2 \\
-0.56914 \pm 2 \\
-0.73030 \pm 3 \\
-0.74047 \pm 3 \\
-0.59383 \pm 3 \\
-0.29641 \pm 2 \\
0.14224 \pm 2 \\
0.72492 \pm 2 \\
1.48991 \pm 2\end{array}$ & $\begin{array}{r}0.0 \\
0.6374382 \pm 9 \\
1.406446 \pm 2 \\
2.423004 \pm 5 \\
3.888328 \pm 8 \\
6.20004 \pm 2 \\
10.23704 \pm 3 \\
18.21009 \pm 6 \\
36.7527 \pm 2 \\
91.8844 \pm 5 \\
346.765 \pm 3 \\
4001.88 \pm 8 \\
-227390 \pm \pm 20 \\
-1139.21 \pm 1 \\
-186.582 \pm 1 \\
-61.3238 \pm 3 \\
-27.8424 \pm 1 \\
-15.49615 \pm 5 \\
-10.04859 \pm 4 \\
-7.33151 \pm 4 \\
-5.77313 \pm 5 \\
-4.64463 \pm 5 \\
-3.55759 \pm 3 \\
-2.31633 \pm 4 \\
-0.72467 \pm 2 \\
0.683817 \pm 3 \\
2.23744 \pm 2 \\
3.69233 \pm 3 \\
5.08168 \pm 3 \\
6.62986 \pm 2 \\
8.845698 \pm 4\end{array}$ \\
\hline
\end{tabular}


TABLE 4

Comparison of Computed Pole Locations for First Painlevé Equation $\lambda=0$

\begin{tabular}{|c|c|}
\hline$\epsilon=0.1$ & Exact \\
\hline $\begin{array}{r}1.2143258 \pm 6 \\
3.64288 \pm 1 \\
6.07142 \pm 4 \\
8.5000 \pm 2\end{array}$ & $\begin{array}{l}1.2143254 \\
3.6429762 \\
6.0716270 \\
8.5002778\end{array}$ \\
\hline \multicolumn{2}{|c|}{$\lambda=1$} \\
\hline$\epsilon=0.1$ & Davis (Approx) \\
\hline $\begin{array}{r}1.2067373 \pm 6 \\
4.4519 \pm 2\end{array}$ & $\begin{array}{l}1.2068 \\
3.0982\end{array}$ \\
\hline \multicolumn{2}{|c|}{$\lambda=5$} \\
\hline $1.1793581 \pm 6$ & 1.179 \\
\hline
\end{tabular}

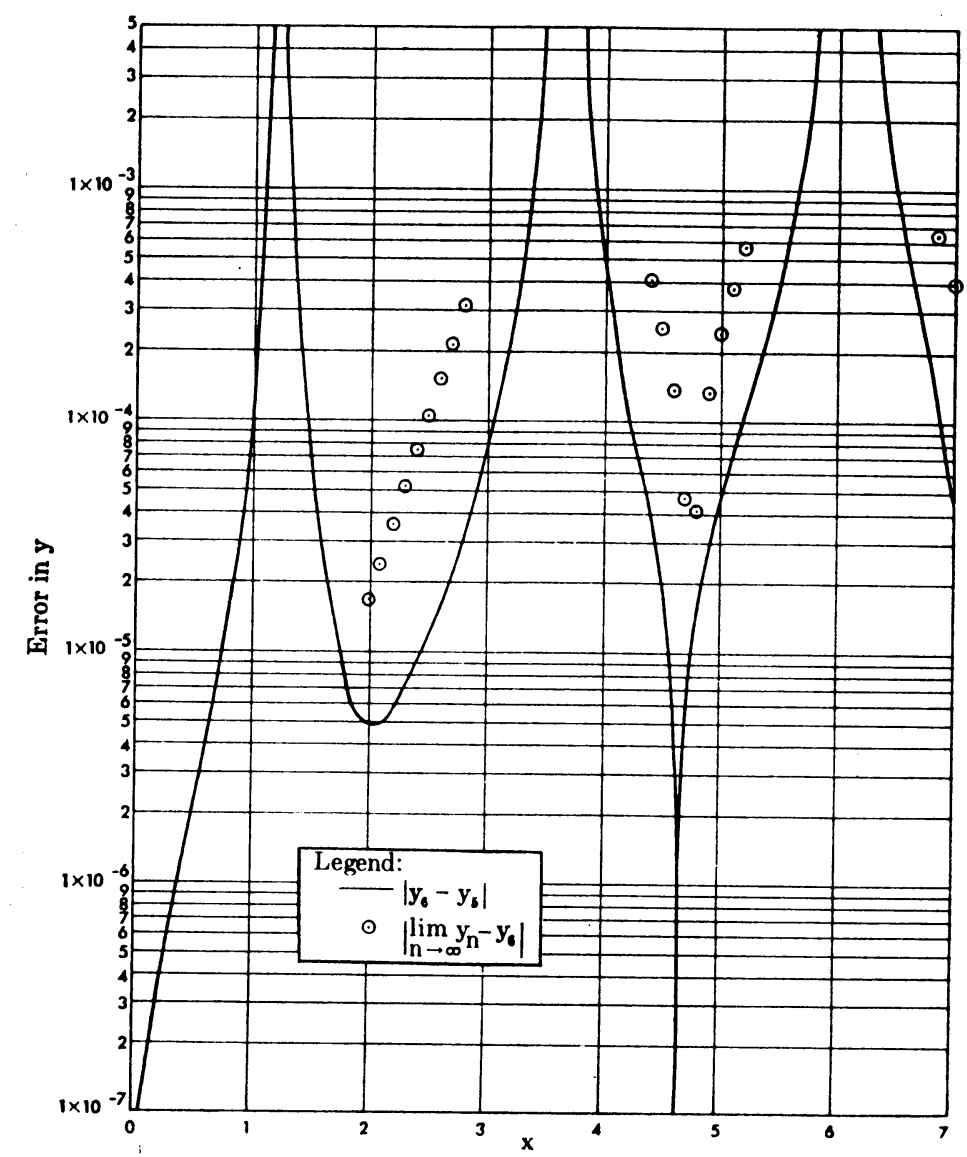

Fra. 1. Comparison of Error Estimate and Actual Error for $\lambda=0(\epsilon=0.1)$ 
case of $\lambda=0$. In this case, the solution is periodic, and it would be possible to obtain the solution for all $x$ 's by translation of the solution in the first period. Since the interest here is in the case where the solution beyond the singularity is not known, and is not necessarily periodic, the numerical solution is continued through the singularities as if its periodic nature were unknown, thus obtaining, by comparison of the numerical solution with the known periodic solution, the actual error of the numerical procedure. This can then be compared with the estimate of error obtained by the numerical computation.

4. Conclusions. A numerical technique for the solution of initial value differential equations whose solutions exhibit movable poles has been proposed. Application to the first transcendent equation of Painlevé has given very satisfactory results.

The Martin Company

Denver, Colorado

1. H. T. DAvis, Introduction to Nonlinear Differential and Integral Equations, U.S. Atomic Energy Commission, U.S. Government Printing Office, Washington, D.C., September, 1960. 